\title{
An Informal Examination of Technological Skills in Library Jobs
}

\author{
by Nina Exner
}

s any librarian can attest, librarianship has always required a wide variety of skills from its professionals. Librarians have always had to cultivate a highly diverse knowledge base to maintain their skills and keep up with the changing requirements of the field. Various discussions have been made about how the librarian's position is evolving and how librarians must seek new skills to grow with it. ${ }^{1}$ Recently, however, technological knowledge and skills have been increasing at a phenomenal rate. In 1996, $66.9 \%$ of academic library jobs required some form of computer skills. ${ }^{2}$ While many traditional skills continue to form the bulk of library job descriptions, one sees technological jargon, acronyms, software names, and so on appearing in library job advertisements with increasing frequency.

This increase of technological terminology can be a source of confusion for people involved in all parts of librarianship. Administrators seeking new systems personnel may have an impression of what competencies they need but lack the proper technological terminology to describe those skills with precision. Librarians wanting to increase their technological knowledge or to change career paths within librarianship can find that they do not have the ability to decipher the jargon well enough to decide which areas to study. Librarians who are al ready techno-savvy may not know how best to keep abreast of the field and hone their abilities. Even in library schools and library assistant programs there is a need to determine the most useful technological skills and begin integrating them into the curriculum.

Technological skills in library job advertisements are becoming more difficult to interpret, rather than easier. Some job advertisements list skills which seem fairly self-descriptive. Other job advertisements are so full of technological jargon that the reader is hard pressed to determine the position's true responsibilities. Job advertisement readers - this author included - may see terms they are unfamiliar with as well as frequently seeing skills listed in ways that confuse them. To help clarify these terms, both for the understanding of job seekers and for the edification of potential job advertisement and job description creators, this article undertakes to define a sampling of recent technological skills sought in the library field. Because this informal examination is not a quantitative analysis, it does not 
enumerate the frequency with which various terms occurred; it merely lists a year's worth of technological job skills and defines and roughly categorizes them with the intention of making a snapshot of the state of the market as well as helping job seekers and job creators to understand those terms.

\section{Methods}

The LIBJOBS mailing list was examined for one year, from December 2002 to November 2003. LIBJOBS is an international mailing list for library jobs, run by the International Federation of Library Associations and Institutions. More information about this mailing list can be found at its home page: http://www.ifla.org/ll/lists/libjobs.htm. Skill lists were copied from sections describing required and preferred applicant qualifications, and mined for all technological, computer, and digital-library-oriented skill descriptions. These skill descriptions were then classified loosely and defined, with links to official sites.

Classifying these skills turned out to be an unexpectedly difficult task. The degree of overlap between areas makes it difficult, if not impossible, to

\section{Acronyms of Skills Required}

ASP - Active Server Pages, a server-side scripting technology that primarily uses the VBScript or JavaScript languages. Like CGI scripting, it describes a method for allowng Web pages to interact with a server.

ASP.NET - Microsoft's Web application development software/environment for the .NET framework. http://www.asp.net/

CFM - ColdFusion Markup files use CFML, ColdFusion Markup Language. ColdFusion is a proprietary development environment for making dynamic Web pages without needing to know a programming or scripting language. This allows the author to do many of the same functions as with server-side scripts.

CGI - Common Gateway Interface, the most common specification used for executing server-side scripts. Perl is the most common language used for CGI scripts, but $\mathrm{C}++$ and Visual Basic can also be used. Server-side scripting is the main method for allowing Web pages to interact with a server in order to do highly dynamic and responsive tasks, including drawing from or writing to a database, composing e-mails, and otherwise responding to online forms and input.

CSS - Cascading Style Sheets are used in conjunction with HTML or other markup languages to control the style and appearance of Web sites. http://www.w3.org/Style/CSS

DC - Dublin Core is one of the most commonly used metadata initiatives. It attempts to be interoperable and broadly functional and adaptable for a variety of uses and purposes. http://dublincore.org

DHTML - Dynamic HyperText Markup Language is something of a misnomer, actually referring more to the process of using HTML, JavaScript, and CSS together and sometimes with other scripting languages to create dynamic effects that HTML alone cannot make.

DOI - A Digital Object Identifier is an identifier similar to a barcode for documents, pictures, and other items of intellectual property in a digital environment. This may come up in "deep linking" of electronic documents in the library catalog to items in online databases. http:// www.doi.org categorize the skills into groups by

function. Therefore, they have only been separated loosely into acronyms and branded or named technologies. There is still a little overlap, of course, and efforts have been made to add references in these cases. Then definitions were added to the terms. Definitions were synthesized from anecdotal personal knowledge, dictionary definitions, and Internet sources. ${ }^{3}$

Furthermore, links to the official site of the standard, software, or technology in question were added where available. The intention is to provide the creator or responsible body's official view of the technology in question, and an attempt was made to use that official information as a main data source whenever possible.

A large number of qualitative skills were also encountered, and considerable thought was given to their best classification and definition. Most job-hunters know that while it is simple to define HTML, it is much harder to know for certain what writers of advertisements mean if they require knowledge of and familiarity with "standard Internet applications." Despite this, many technology advertisements, especially for systems supervisors, concentrated on these more descriptive statements. Therefore they have been synthesized and included at the end of the article without attempt at definition or explanation. 


\section{Results}

A year's worth of job advertisements on the LIBJOBS listserv yiel ded a total of 194 postings, running from November 2002 to December 2003. This represented a total of 206 job openings examined in the data-mining process.

Examination of the skills reveals a very broad range; technological skills are so widely needed in the library field that they can be found in all arenas. Many technological skills were required not only in the everincreasing number of systems jobs but in public and technical services areas as well.

It is worth noting that skills

\section{Acronyms continued}

DTDs - Document Type Declarations are files that have been written to interpret $\mathrm{XML}$ and other extensible languages.

EAD - Encoded Archival Description is a metadata format intended for describing archival information and materials. http://www.loc.gov/ead/

FGDC - The Federal Geographic Data Committee produces metadata standards on geospatial information. http://www.fgdc.gov/metadata/ meta stand.html

HTML - HyperText Markup Language is the most common method of preparing text for display on the Web. Many advertisements specify proficiency in "handcoding" HTML, which means being able to mark up HTML documents by hand rather than creating them in an HTML editor.

IIS - Internet Information Server is Microsoft's operating system software for running a Web server.

LAN - Local Area Network.

LDAP - Lightweight Directory Access Protocol is a network protocol for retrieving information from directories.

LOM - Learning Object Metadata is a metadata system for assigning attributes to describe things relevant to educational technology. http:// Itsc.ieee.org/wg12/

MARC - MAchine-Readable Cataloging is the format for storing library catalog records for use by Online Public Access Catalogs. http:// www.loc.gov/marc/

Marc21 - The current standard of MARC.

METS - Metadata Encoding and Transmission Standard is an XML-based standard from the Library of Congress, used for encoding metadata regarding objects within a digital library. http://www.loc.gov/standards/ mets/

NITF - News Industry Text Format is an XML-based system for defining the structure, content, and metadata of news articles. http://www.nitf.org

OAI or OAI-PMH - Open Archives Initiative for Metadata Harvesting Protocol is the format in which OAl-participating institutions should encode their documents. http://www.openarchives.org/OAl openarchives protocol.html frequently described as being in one category by the employer would be more accurately classified in another. For example, metadata skills were grouped with Web skills in some advertisements; it was unclear whether they were intentionally being mixed together. This confusion simply reinforces the impression that job description writers and job search committees may need better understanding of the skills for which they are searching.

Over fifty items were identified for definition and explanation. These ranged widely, from highly specific skills (such as programming languages, specific applications, network configurations, and operating systems) to broader topics (such as familiarity with standards and general procedures). Online Public Access Catalogs (OPACs) by specific brand name were excluded, however. Suffice it to say that if OPAC administration knowledge was required for a position, generally preference was given to candidates who knew the in-house system.

\section{Discussion}

Though the primary goal of this paper was to provide concise definitions of currently sought-after skills to promote a clearer understanding for job seekers and job description writers alike, it is worth discussing these skills somewhat. In examining the list one can see what skills were in demand during this time period.

Not surprisingly, some of the most commonly required softwarespecific skills were proficiency in the use and support of Windows terminals and knowledge of Microsoft Office suite applications. Even jobs 
which otherwise would not be described as particularly technological often mentioned Microsoft Office products such as Word, Excel, and PowerPoint. Croneis and Henderson found that between 1990 and 2000, 18.8\% of computer-oriented library jobs required program or technical support. ${ }^{4}$ This figure, however, does not include the number of non-computer oriented librarians who also perform program and technical support tasks. Other studies found that by

Acronyms continued

OCR software - Optical Character Recognition software is used to convert scanned documents to text.

ONIX - ONline Information eXchange is a standard, XML-based format for storing and transmitting information about books, used by the publishing and bookselling industries. http://www.editeur.org

OpenURL - The Open Uniform Resource Locator standard is for encoding Web-transferable metadata and identifiers for information on the Internet to create URLs that are context-sensitive. http://www.niso.org/committees/ committee ax.html

OSI - 1) Short for ISO/OSI, the International Organization for Standards Open Systems Interconnect, the model which defines the seven layers of types of interrelated interactions in a network, a fundamental concept in networking. 2) Open Source Initiative is an organization which promotes free distribution of software, along with the source code (the uncompiled program, for people to see how it was written).

PHP - PHP Hypertext Preprocessor is a scripting language used mostly for server-side scripting that can be embedded in HTML pages. It is gaining popularity versus other server-side scripting methods. http://www.php.net

PURL - Persistent Uniform Resource Locators point to an intermediate resolution service which associates the PURL with the actual URL. PURLs are a project of OCLC's research division in collaboration with other groups. http://purl.org

SGML - Standard Generalized Markup Language is an ISO standard for markup languages.

SQL - Structured Query Language is the most common language for making database queries to retrieve information from a database.

TCP/IP - Transmission Control Protocol / Internet Protocol is a set of protocols for computers to communicate over a network. Network administrators have to know how to configure computers' TCP/IP settings.

TEI - Text Encoding Initiative produces a standard for encoding text, primarily in the humanities, in digital format. http://www.tei-c.org/

VRA Core - Visual Resources Association Core Categories is a metadata system for describing and encoding works of art and images. http:// www.vraWeb.org/vracore3.htm

XML - eXtensible Markup Language is a flexible system for creating markup for documents and their associated metadata, geared towards use on the Web. http://www.w3.org/XML/

Z39.50 - Z39.50 refers to the International Standard, ISO 23950: Information Retrieval (Z39.50): Application Service Definition and Protocol Specification. http://www.loc.gov/z3950/agency/ the late 1990s computer technolo-

gies had become routine across the board in academic libraries. ${ }^{5}$ Office suite use and support could be considered one of the most fundamental such skills likely to be needed.

Of particular interest is the burgeoning field of metadata and digital library skills. These skills were widely in demand for a variety of areas outside of the system department, particularly cataloging and archives. Image processing and scanning experience were often linked to metadata knowledge in the archives areas, whereas traditional cataloging abilities and MARC knowledge were unsurprisingly emphasized in metadata cataloging positions. Systems jobs describing metadata skills tended to group them with Internet skills and Web authoring knowledge. Metadata and digital-library-related skills were numerous; this is noticeably a growing field of interest.

Server and network administration skills tended to be more broadly and qualitatively described. A few jobs had several very specific protocol and process descriptions, but most preferred to describe skill sets in general terms such as "Windows network technology," or "client/server architecture."

Web coding skills (HTML or a page authoring program of some kind) were al so asked for frequently. Basic, sometimes very basic, familiarity with Web page creation seems to have become ubiquitous as a skill requirement among library job applicants in public services. However, the ever-evolving world of Web design may be reflected in the fact that there was not as a wide variety of Web skills as might have been imagined. Though a great many Web skills were listed in job advertisements, there were also many 


\section{Brand Names/Technological Terms}

Adobe Photoshop - A graphics processor for all purposes http:// www.adobe.com/products/photoshop/main.html

Checkpoint - A security and firewall system http://www.checkpoint.com

DSpace - DSpace is a digital library or repository that helps capture, store, index, preserve, and redistribute research documents and output. http:// www.dspace.org/

EZProxy - A user authentication system http://www.usefulutilities.com

Fireworks - A graphics processor, particularly for Web graphics http:// www.macromedia.com/software/fireworks/

Flash - An online video creation program http://www.macromedia.com/ software/flash/

ILLiad - OCLC's software for sharing interlibrary loan documents with other libraries using ILLiad http://www.oclc.org/illiad/

Java - A programming language for writing programs on and off the Web, not to be confused with and not generally interchangeable with client- or server-side scripting languages. http://java.sun.com

JAVApplets/Javascripting - JAVApplets are programs written in the Java language, and JavaScripts are usually client- side scripts for manipulating HTML and interacting with users.

JavaScript - A scripting language, mostly though not always used for creating quick client-side scripts to make dynamic Web page effects.

Linux servers - An open source operating system for workstations or networks/servers, available in several different distributions or versions.

Macromedia Dreamweaver - A Web-authoring program http:// www.macromedia.com/software/dreamweaver/

Microsoft FrontPage - A Web-authoring program http://www.microsoft. com/frontpage

MSOffice - A productivity suite (Word, Excel, PowerPoint, etc.) http:// office.microsoft.com

Novell network - A networking operating system http://www.novell.com

Perl - A programming/scripting language, often used for writing server-side scripts via CGI

Shibboleth - A user authentication system http://shibboleth.Internet2.edu/

Sun/Solaris - Solaris is the operating system used on Sun Microsystem's SPARC and $x 86$ servers. http://wwws.sun.com/software/solaris/

Unix - An operating system. Unix and Linux systems (which are closely related but not the same) together account for the majority of Web server space on the Internet.

Windows NT - An operating system, mainly for networked computers. http://www.microsoft.com/windows/

Windows 2000 - An operating system, mainly for networked environments. http://www.microsoft.com/windows/ skills, programs, and page authoring environments that were not listed.

This might indicate that libraries are all using the same few authoring tools, or it may simply mean that many job creators are content to let most tools and standards fall under the heading of broad descriptors such as "accessibility issues" and "Web development tools."

This leads to something of a digression. A great deal of confusion continues to exist about programming and scripting for Web pages. Authors of advertisements for Web developers may have some misapprehensions about the roles of different Web languages. Java and JavaScript, which are very different tools, are often confused and lumped together. In fact, it is not unusual to see a variety of basically unrelated skills such as HTML, XML, CGI, and "Java/JavaScript" grouped together in a listing as alternatives to each other (for example, "candidates must have one or more of these skills"). Java is made for writing full programs, which can be executed either within or outside of a Web browser. Java is not generally used for making simple Web page effects and elements, unlike JavaScript. Java and JavaScript, while often confused by non-technological personnel, fill two very different roles. Tools for Web markup, client-side scripting tools, server-side scripting tools, and programming languages simply are not comparable in the Web design world. Adding skills like XML, which is properly thought of in relation to metadata rather than Web pages, though it actually overlaps both categories, merely confused the issue. As a guideline, client-side tools, such as JavaScript, are used for making interesting dynamic page effects, as well as useful but simple tasks such as browser validation. Server-side tools like CGI, PHP, some ASP, and ColdFusion, are intended to allow the Web page to interact with a server. This includes writing to a database, generating response pages, creating e-mail messages, and so on. Style controllers like CSS and XSL 


\section{Broad Categories of Skills Required}

Administrative and Interdisciplinary

- Budgeting

- Contracts and licenses; licensing agreements

- Copyright issues

- Evaluation of electronic resources

- Familiarity with library automation products (especially portal concepts, desk-top computing and Web applications)

- Hardware and software purchasing

- Programming and application development; some programming experience

- Project management experience

Digital Libraries / Metadata / Archiving

- Awareness of the issues involved in archiving electronic resources

- Broad knowledge of current practices in digital library development

- Demonstrated knowledge of the principles, practices, and techniques of archival descriptive standards

- Digital rights management

- Familiarity with digitization production processes and standards

- Knowledge of digital imaging management formats

- Relevant digital library issues, trends, and standards, including metadata

- Scanning and imaging

Instruction

- Electronic classroom teaching experience

- Trends in instructional technology

Technical Support and Networking

- Client/server computing architecture

- Computer skills in a PC/Windows environment

- Integrated library systems

- Knowledge of relational database management systems

- Network technology

- Networking and data communications technology

- PC support

- Understanding of data communications protocols, software and networks

Web Authoring

- Accessibility issues

- Demonstrated experience with the design, development, and management of Web sites, including HTML authoring, form creation, editing/ site management software

- Demonstrated working knowledge of database-driven Web sites

- Experience with online bibliographic tools, Web database software, Web design

- HTML authoring, form creation, editing/site management software

- Internet and Web-based information tools

- Log analysis, graphic editing tools

- Previous experience in graphic design

- Standard Internet applications

- Technical issues associated with electronic publishing and Internet development

- Thorough knowledge of CSS and the use of templates and library items in Dreamweaver

- Thorough understanding of Web design principles and techniques

- Usability testing

- Web and server administration experience

- Web authoring tools are used to control details of the appearance of Web pages in ways markup alone cannot. All of these still depend on the basic tool of Web page authoring, HTML or XHTML which make the page display in the browser-readable format. When writing an employment advertisement, it is preferable to use broad skill descriptions and to indicate that knowledge of certain categories of authoring, such as server-side scripting or handcoding of dynamic Web pages, are required by applicants.

Original programming and software development skills were rather rare as requirements, outside of specialized areas, including Web and database programming skills. This is probably reflective of the increasing number of computer professionals in libraries to deal with such specific tasks.

Qualitative skill descriptions were very common, and ranged widely. Some were clear, descriptive, and well articulated. Others were either very broad or very vaguely phrased, leaving the reader puzzled as to the exact skills being sought. Even a simple phrase such as "Windows XP," if unaccompanied by an explanation, might imply anything from basic familiarity with the use of the operating system, to in-depth troubleshooting skills, to XP network administration.

In all areas, computer skills showed considerable mixing. All sorts of technological skills were observed in all subsets of library jobs, within all parts of public services, technical services, administrative positions, and systems positions.

\section{Conclusion and Suggestions for Future Research}

Technological skills have become ubiquitous in librarian positions. It is almost pointless to attempt to separate technological from nontechnological library jobs; all library jobs have become technologically oriented to the point that they require a level of proficiency with at least some programs. Whether in systems, administrative, technical 